\title{
Belt Feeder for Biomass Mixing
}

\author{
Aivars Kaķītis, Imants Nulle, Dainis Ancāns \\ Latvia University of Agriculture, Faculty of Engineering, Institute of Mechanics \\ Address: J. Cakstes blvd. 5, Jelgava, LV-3001
}

\begin{abstract}
Level of agricultural productivity has been increased and also increased land area not utilized for food production. This area can be used for growing energy crops, including grasses. In addition, there are non-agricultural lands in Latvia suitable only for growing energy crops like Reed canary grass etc. Reed canary grass growing for energy needs could be an additional source of income for Latvian farmers, whom have harvesting technique for hay, for example dairy farming. It could be also a local heat energy source if wood in farm area is not available. Pelletizing or briquetting of dried herbaceous biomass has several advantages such as increasing energy density, improving storability and reducing handling and transport costs, but remains, of the fuel quality negatively affecting things, such as high ash and chlorine content (wheat straw $0.1-2.3 \%$ ). Therefore, to reduce ash content and corrosion of heat transfer surfaces suggestible to make blends with others biomass where those parameters are lower. Briquettes of herbaceous biomass blends with peat are denser, durabler and take less energy for briquetting and pelleting. A new mixing method in this article is described. Experiments of biomass feeding in mixing process with belt feeder were done. The throughput of the belt feeder is increasing nearly linearly at feeder belt velocity from 0.18 to $0.89 \mathrm{~m} \cdot \mathrm{s}^{-1}$. The specific throughput of belt feeder was evaluated for Reed canary grass. Noticeable influence of the belt velocity on the specific throughput of belt feeder was not found.
\end{abstract}

Keywords - mixer, feeder, Reed canary grass.

\section{INTRODUCTION}

Biofuels and especially - herbaceous biomass, contain more alkali metals (mainly potassium and sodium) than coal and peat. Alkali metals lower the ash melting point and upon reaction with chlorine, also contained in biomass, have a strong corrosive effect on heat exchangers [1]. Strategies for minimizing deposition problems include co-firing biofuels with "cleaner" fuels. Suitable "clean" fuels include certain types of coal or peat, which contain large amounts of inert species such as silica or alumina in their ash. By blending peat with chips or herbaceous biomass also sulphur content of the fuel is increased, sulphates are formed instead of chlorides, and the risk of corrosion is avoided [2].

Components of mixtures have to be in the required proportion and homogeneous mixed to provide good quality (density, durability, and burning properties) of briquettes or pellets. Usually in mixing process at least two feeders and mixer have been involved. If for bulk material feeding two belt feeders are used, blends from two components can be achieved by merging flows without additional mixing [3]. This type of inflow mixing showed good results. The standard deviation of the component field ratio was $6 \%$ that is an acceptable result for biomass mixtures [3].

To achieve better results it is necessary additional mixing but it requires for additional technique unit. The task of the research was to design biomass mixer without additional expensive components. Therefore the mixer showed in Fig. 1 was designed. This mixer is recommended for mixing different kinds of bulk materials (Patent Nr. LV14603 B). Mixer consists of two belt feeders with special knobby belts. Feeders are placed above each other in determined height and belts are moving with appropriate speeds to ensure additional mixing of two components in space between conveyor belts (Fig. 2).

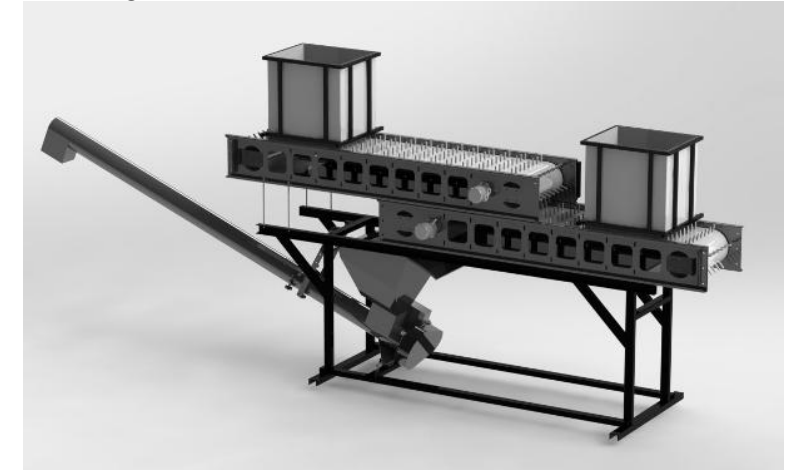

Fig. 1. Bulk material mixer (Patent Nr. LV14603 B)

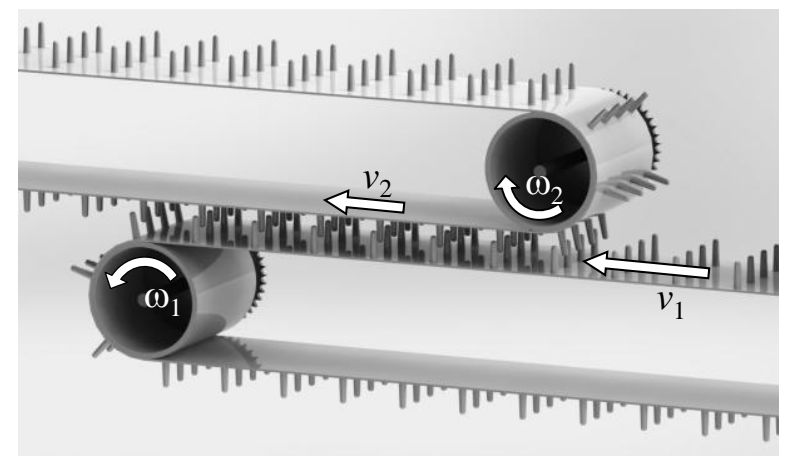

Fig. 2. Working principle of mixer

Mixing process is essentially influenced by quality of the continuous feed. The objective is to feed set quantities of material per unit of time in an uninterrupted product flow. Feeding can be performed volumetrically by feeder equipment which draws material by volume or gravimetrically by controlled feeding using weight or mass as the control value. Gravimetric feeding offers greater accuracy even over 
periods of hours or days (long term constancy) and is also suited to the feeding of materials with fluctuating bulk density or flow properties, such as cohesive powders and liquid additives with a variable viscosity.

Gravimetric feeding also enables the actual metered volume to be reported back for the purposes of recording, taking printouts and storing, as well as data transference to the process control, information management and alarm systems [4].

By feeding volumetrically it is important that the flow pattern be such that the whole outlet of the feed hopper is fully active. This is of fundamental importance in the case of mass-flow hoppers [5]. In a mixer design presented in this paper (Fig. 1) feed hoppers outlet is fully active. In this case respect of this rule is very important because of poor flowability of the chopped biomass. Mixer should have to have possibility to change proportion of blended components therefore feeder throughput characteristic is important to calculate parameters and to adjust feeders. In this paper the volumetric throughput of a belt feeder by feeding Reed canary grass was stated.

\section{MATERIAL AND METHODS}

Estimation of the volumetric throughput of a belt feeder was carried out in experimental equipment (Fig.3). It consists of belt feeder, bin for chopped biomass with changeable gate opening and hydraulic motor for driving feeder.

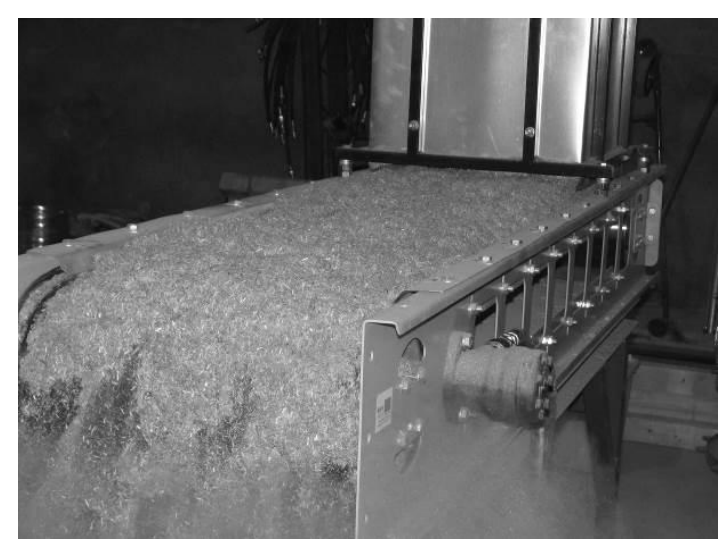

Fig. 3. Experimental equipment

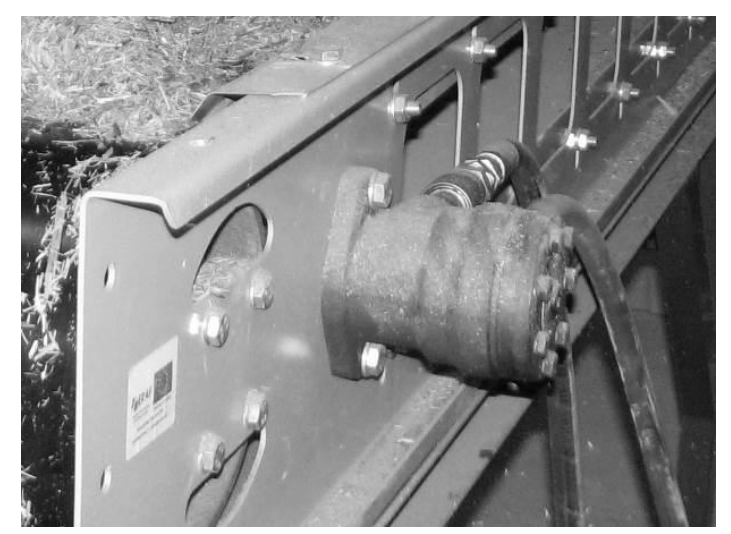

Fig. 4. Hydraulic drive of feeder
Rotation frequency of feeder was changed by hydraulic motor (Fig. 4). The angular velocity of feeder was measured with inductive sensor and impulse counter MP5W-4N (Fig. 5). Feeder bin was filled with determined weight chopped Reed canary grass biomass. Time was set during bin emptying.

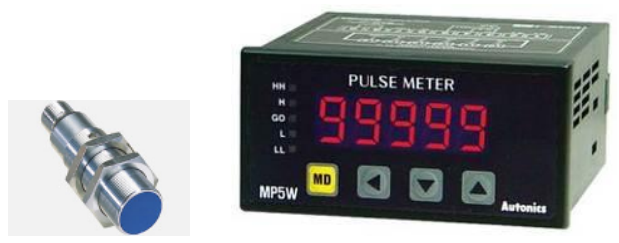

Fig. 5. Inductive sensor and impulse counter

Experiments were carried out with chopped Reed canary grass. There were two experiment sets with different particle size distribution of chopped biomass (Fig. 6 - coarse chopped and Fig. 7 - fine chopped). Moisture content of Reed canary grass was $\sim 22 \%$.

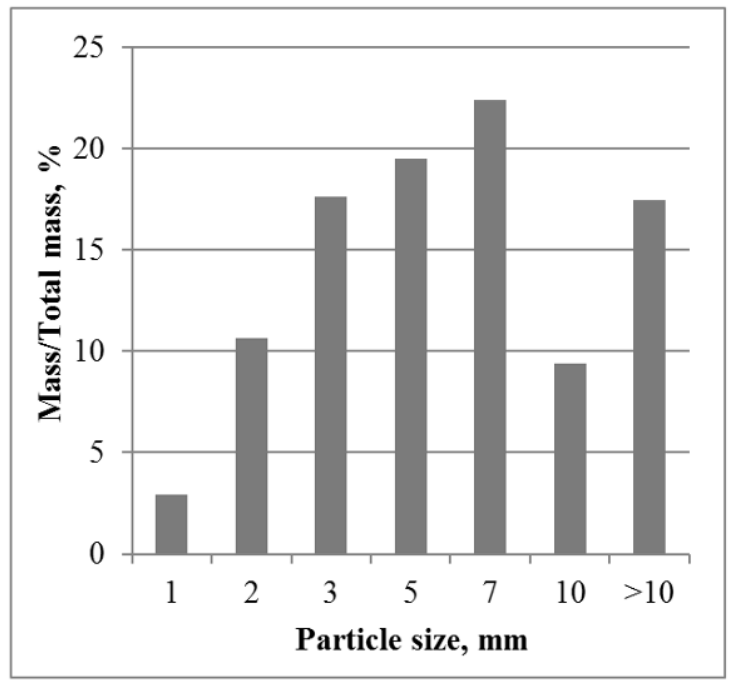

Fig. 6. Particle size distribution of chopped material for first experimental set - coarse chopped

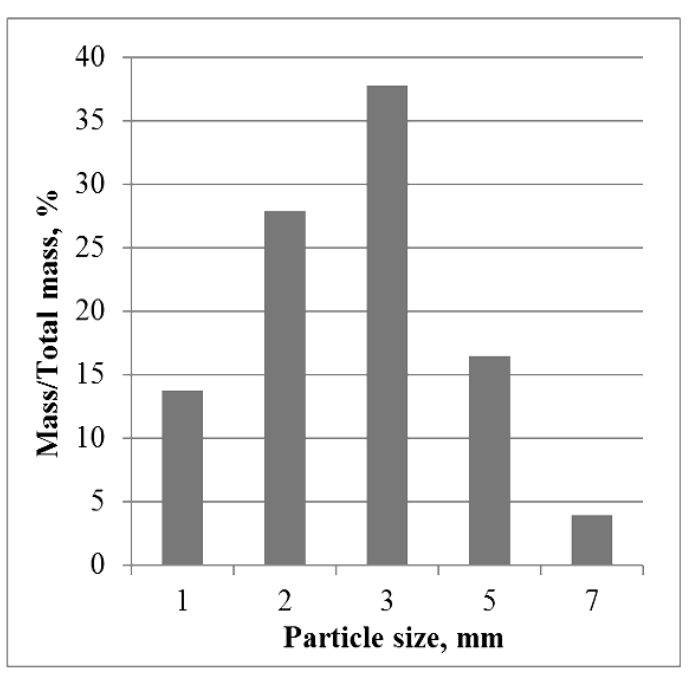

Fig. 7. Particle size distribution of chopped material for second experimental set- fine chopped 
In the first experiment set coarse chopped biomass was used. Sieve analyze showed that there are more than $15 \%$ particles that didn't pass through sieve with holes diameter $10 \mathrm{~mm}$. Visual estimation (Table 1) of particles let us state that particles of chopped stalk are thin and long. Particles passed through sieve holes with diameter $3 \mathrm{~mm}$ could be in average $5-10 \mathrm{~mm}$ but in some cases up to $30 \mathrm{~mm}$ long.

In the second experiment set fine chopped particles was used there were just $4 \%$ of particles which didn't passed through sieve with $5 \mathrm{~mm}$ holes.

TABLE I

SIEVE AND PARTICLES SIZE

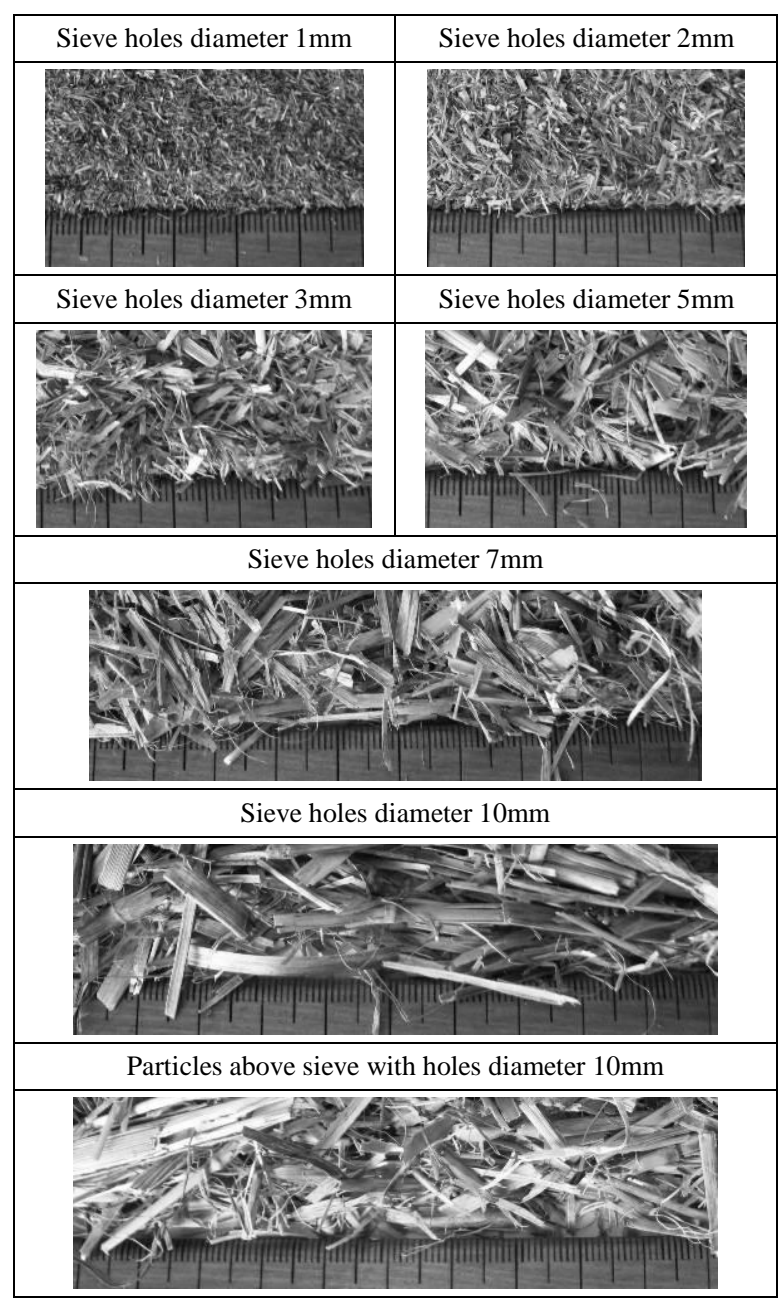

\section{RESULTS AND DISCUSSION}

Experiments were carried out to establish parameters of belt feeder by feeding Reed canary grass. The throughput of the belt feeder is increasing nearly linearly at feeder belt velocity from 0.18 to $0.89 \mathrm{~m} \cdot \mathrm{s}^{-1}$. The throughput with rough chopped particles and bin gates opening $20 \mathrm{~mm}$ grows from 0.02 to $0.11 \mathrm{~kg} \cdot \mathrm{s}^{-1}$ (Fig. 8) but for fine from 0.08 to $0.33 \mathrm{~kg} \cdot \mathrm{s}^{-1}$ (Fig. 9). The feed intensity is influenced by particles size, particles orientation and other bulk properties. Bulk density of fine chopped particles is higher and also throughput is higher. There are some other factors that influences feeder throughput. Coarse chopped Reed canary grass is a material with poor flowability. In Fig.
12 is showed that material is pulled out from the bin with $\mathrm{V}$ design profile belt. As a result is discontinuous flow. Even at large opening $75 \mathrm{~mm}$ the flowabilty of material is still poor. Moreover, the material itself makes jam (Fig. 13) decreasing opening and feeder throughput. After some time jams collapses and creates blowouts (Fig. 14) therefore material flow stability brakes down.

Belt feeder throughput increases to $1.8 \mathrm{~kg} \cdot \mathrm{s}^{-1}$ at belt velocity $0.89 \mathrm{~m} \cdot \mathrm{s}^{-1}$ and opening $75 \mathrm{~mm}$ (Reed canary grass fine chopped) (Fig. 10)

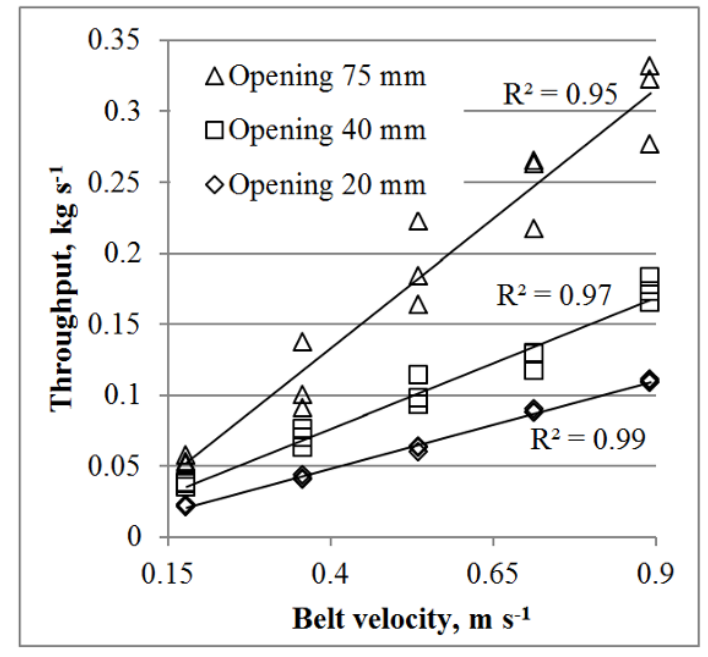

Fig. 8. Feeder throughput with coarse chopped Reed canary grass

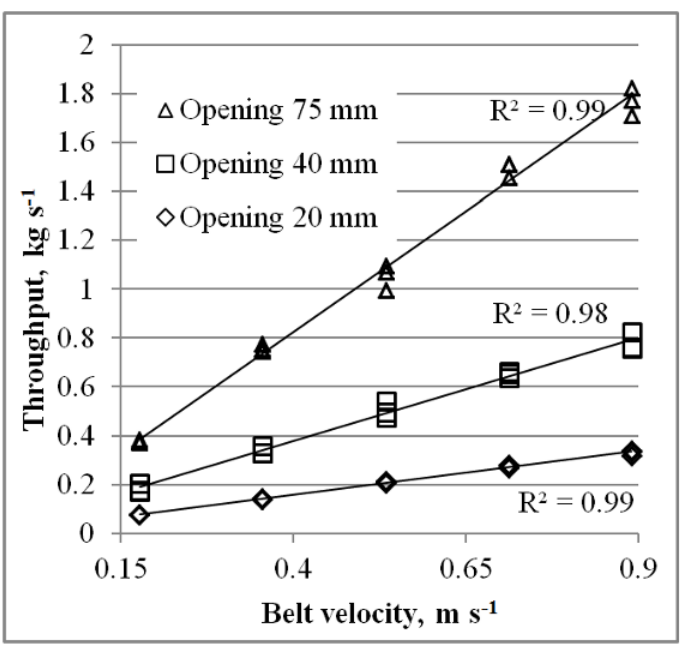

Fig. 9. Feeder throughput with fine chopped Reed canary grass 
Kak̄īis A., Nulle I., Ancāns D. BELT FEEDER FOR BIOMASS MIXING

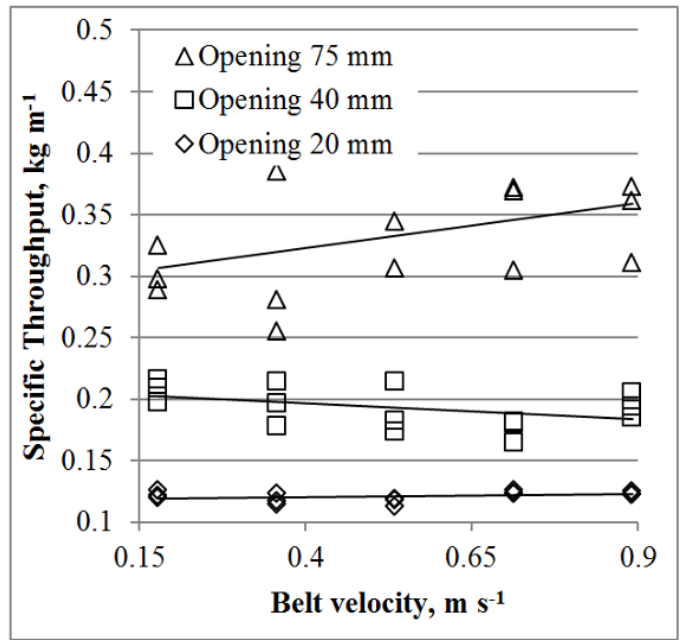

Fig. 10. Specific throughput of the belt feeder with coarse chopped Reed canary grass

The specific throughput of belt feeder shows that there is no noticeable influence of belt velocity on it by feeding coarse chopped particles (Fig. 10). The specific throughput at opening 20,40 and $75 \mathrm{~mm}$ respectively is $0.12 \pm 0.002, \quad 0.19 \pm 0.009, \quad 0.33 \pm \quad 0.025 \mathrm{~kg} \cdot \mathrm{m}^{-1}$ (Confidence Level 95.0\%).

The specific throughput of finer particles by the same belt speed is higher (Fig. 11) because of the density of material and steady flow (Fig. 3). The specific throughput at opening 20,40 and $75 \mathrm{~mm}$ respectively is $0.39 \pm 0.011, \quad 0.94 \pm 0.041, \quad 2.06 \pm$ $0.047 \mathrm{~kg} \cdot \mathrm{m}^{-1}$ (Confidence Level $95.0 \%$ ).

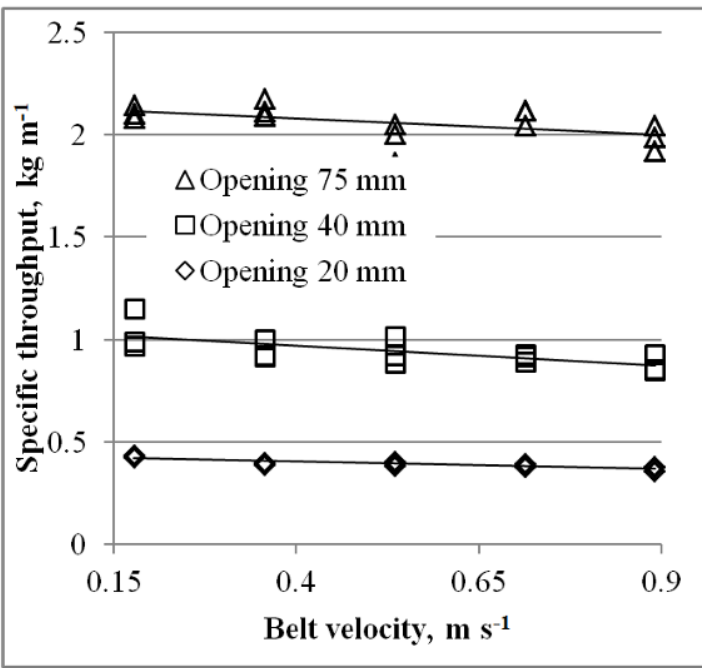

Fig. 11. Specific throughput of belt feeder for second set of experiment

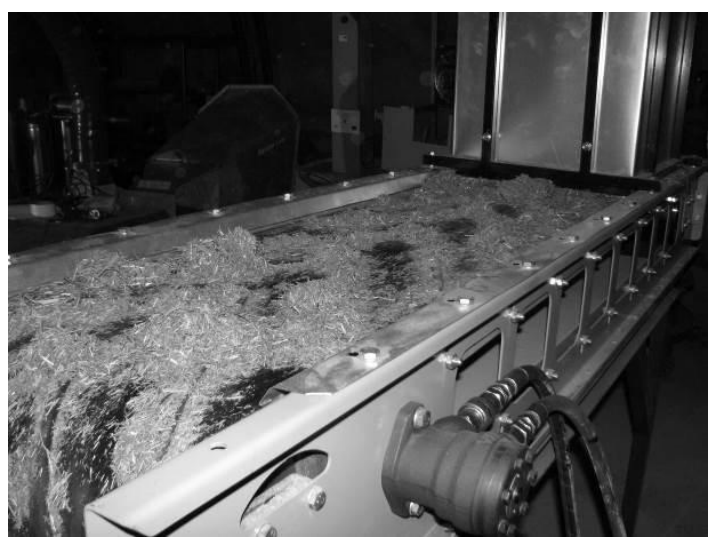

Fig. 12. Discontinuous flow

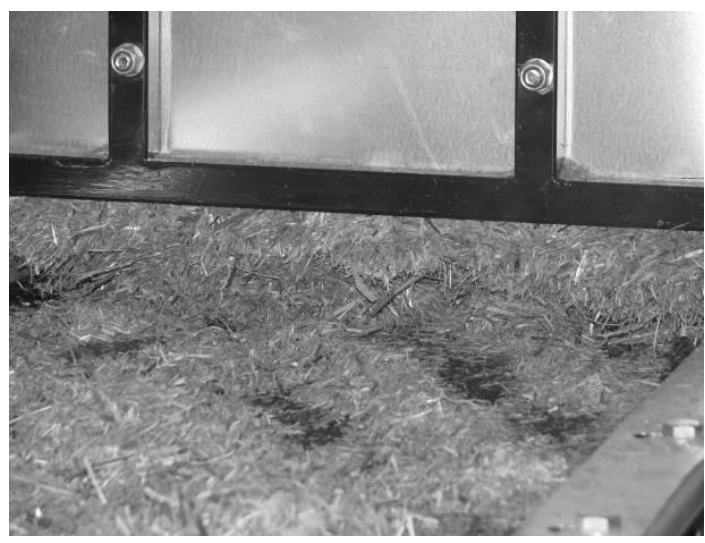

Fig. 13. Jam of chopped biomass

Belt feeder application for fine chopped Reed canary grass is acceptable. Necessary to continue experiments of feeder equipped with special knobby belts showed in Fig. 2.

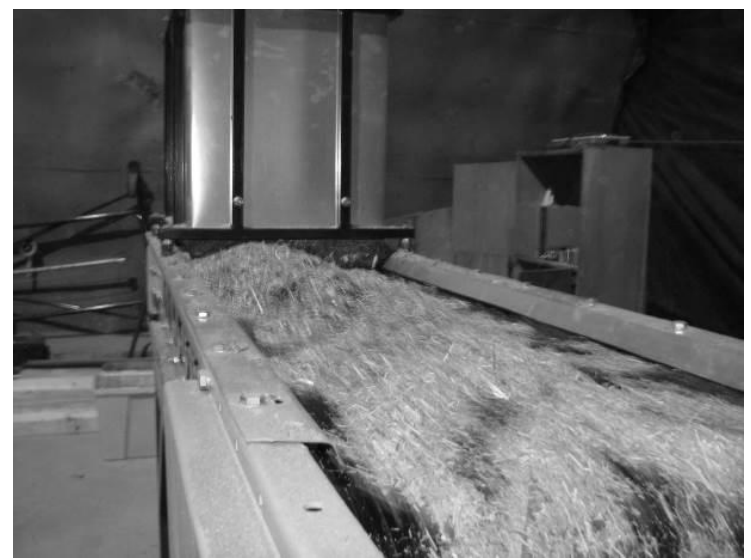

Fig. 14. Blow-out

\section{CONCLUSIONS}

1. The throughput of the belt feeder is increasing nearly linearly at feeder belt velocity from 0.18 to 0.89 $\mathrm{m} \cdot \mathrm{s}^{-1}$ and it grows from 0.02 to $0.11 \mathrm{~kg} \cdot \mathrm{s}^{-1}$ (opening 20 $\mathrm{mm}$, rough chopped biomass).

2. Belt feeder throughput increases to $1.8 \mathrm{~kg} \cdot \mathrm{s}^{-1}$ at belt velocity $0.89 \mathrm{~m} \cdot \mathrm{s}^{-1}$ and opening $75 \mathrm{~mm}$ (Reed canary grass fine chopped). 
3. Belt feeder application for fine chopped Reed canary grass is acceptable. Necessary to continue experiments of feeder equipped with special knobby belts

\section{$\mathrm{V}$ ACKNOWLEDGMENTS}

This publication has been prepared within the framework of the ERAF Project „Development of mechanisation equipment for energy crops conditioning", contract

Nr. 2010/0306/2DP/2.1.1.1.0/10/APIA/VIAA/128.

\section{REFERENCES}

[1] B. Kavalov, D. Peteves. Bioheat applications in the European Union: An analysis and perspective for 2010. Luxembourg: Office for Official Publications of the European Communities, 2004. 122 p. ISBN 92-894-8730-5.

[2] E. Alakangas, D. Agar, M. Leppänen. Report of the seminar presentations and study tour [online]. Biomass Logistics and Combustion [viewed 2013-01-15]. Accessible: http://www.opetchp.net/download/wp3/summerschoolreport. pdf

[3] A. Kakītis, I. Nulle. Electrostatic Biomass Mixing. In: Engineering for rural development.: Proceedings of 8th International Scientific conference Latvia University of Agriculture, Faculty of Engineering. Jelgava: LLU, 2009, p. 247 -252 .

[4] R. Weinekötter, H. Gericke. Mixing of solids. Dordrecht: Kluwer Academic Publisher. 2000. 151 pp.

[5] A.W. Roberts. Design and application of feeders for the controlled loading of bulk solids onto conveyor belts. [viewed 2013-02-9]. Accessible: http://www.saimh.co.za/beltcon/ 